\title{
Polymer Waveguide Coupled Surface Plasmon Refractive Index Sensor: A Theoretical Study
}

\author{
Lanting JI, Shuqing YANG, Rongna SHI, Yujie FU, Juan SU, and Chi WU* \\ Institute of Marine Science and Technology, Shandong University, Qingdao 266237, China \\ *Corresponding author: Chi WU E-mail: qi.wu@sdu.edu.cn
}

\begin{abstract}
A waveguide coupled surface plasmon sensor for detection of liquid with high refractive index (RI) is designed based on polymer materials. The effects of variation of the thickness of the Au film, polymethyl methacrylate (PMMA) buffer, and waveguide layer on the sensing performance of the waveguide are comprehensively investigated by using the finite difference method. Numerical simulations show that a thinner gold film gives rise to a more sensitive structure, while the variation of the thickness of the PMMA buffer and waveguide layer has a little effect on the sensitivity. For liquid with high RI, the sensitivity of the sensor increases significantly. When RI of liquid to be measured increases from 1.45 to 1.52 , the sensitivity is as high as $4518.14 \mathrm{~nm} / \mathrm{RIU}$, and a high figure of merit of 114.07 is obtained. The waveguide coupled surface plasmon RI sensor shows potential applications in the fields of environment, industry, and agriculture sensing with the merits of compact size, low cost, and high integration density.
\end{abstract}

Keywords: Waveguide; surface plasmon polaritons; polymer; refractive index sensor

Citation: Lanting JI, Shuqing YANG, Rongna SHI, Yujie FU, Juan SU, and Chi WU, "Polymer Waveguide Coupled Surface Plasmon Refractive Index Sensor: A Theoretical Study," Photonic Sensors, DOI: 2020, 10(4): 353-363.

\section{Introduction}

As one of the most important physical parameters of liquid, refractive index (RI) reflects the essential characteristics of liquid and is closely related to temperature, concentration, density, and thermal coefficient. Therefore, in the field of physical, chemical, biological, medical, environmental research and production practice, the monitoring of liquid RI variation is more and more important. Surface plasmon polaritons (SPPs) are electromagnetic waves coupled with free electron density oscillations that propagate along the interface between a metal and a dielectric medium [1-3]. The mode field distributions of the SPP mode are highly localized in the vicinity of the metal surface and exhibit significant response to the variation of external RI near the metal. SPP-based sensors have been widely used in food safety, medical diagnosis, and biochemical sensing fields with merits of high sensitivity, label-free, and real-time detection [4-13]. Due to the mismatch of propagation constants, the SPPs cannot be excited directly by light waves. Many excitation configurations have been reported, in which Kretschmann or Otto prisms [14-16], gratings [17], waveguides [18], and fibers [19] are popular ones. A variation of the RI of medium will produce a change in the propagation constant of the SPP waves that alters the coupling condition between the light waves and SPP waves. Most of plasmonic sensors utilize Kretschmann or Otto prisms to excite SPP

Received: 15 November 2019 / Revised: 23 April 2020

(C) The Author(s) 2020. This article is published with open access at Springerlink.com

DOI: $10.1007 / \mathrm{s} 13320-020-0589-\mathrm{y}$

Article type: Regular 
waves based on attenuated total reflection $[4,6,14$, 16]. These are large in volume, high in cost, and difficult to integrate and miniaturize. The evanescent field coupling waveguide offers the benefits of small size, compact sensing elements, and potential of multichannel sensors on a chip. Various waveguide-type surface plasmon resonance (SPR) sensors such as the slab waveguide, channel waveguide, and even more complex waveguide structures have been demonstrated [20-26]. By optimizing the waveguide parameters, the interaction between the light waves and SPPs waves can be better controlled to realize a better sensing performance. Fang designed an RI sensor based on coupling between the long range surface plasmon polariton mode and the dielectric-waveguide mode, showing a high RI resolution of $4 \times 10^{-7}$ refractive index unit (RIU) [27]. Fan et al. reported a vertical coupler consisting of surface plasmon waveguide and dielectric waveguide, and realized an RI resolution of $7.3 \times 10^{-6} \mathrm{RIU}$ and ultra-thin layer sensing with a detection sensitivity of $0.67 \mathrm{~dB} / \mathrm{nm}$ [28]. A polymer waveguide SPP based RI sensor was fabricated in our previous work, and an RI resolution of $1 \times 10^{-6}$ RIU was obtained [29]. These waveguide-type SPR sensors based on optical power detection are favorable to monolithic integration and on-chip sensor. However, their performance is highly affected by the instability of the light source and the in/out coupling efficiency of the waveguide. Ma et al. reported a long-range surface plasmon waveguide (LRSPP) coupled with the dielectric waveguide biosensor [8]. By optimizing the waveguide parameters, the sensitivity is as high as $4180 \mathrm{~nm} / \mathrm{RIU}$. Satyendra et al. proposed an RI sensor made of a polymer channel waveguide coated with a copper film and $10 \mathrm{~nm}$ silicon-dioxide protecting layer, and a maximum RI sensitivity of $\sim 45 \mu \mathrm{m} / \mathrm{RIU}$ was obtained [30]. These works are based on the spectral interrogation. Compared with the power detection, spectral interrogation has a higher stability and accuracy. Due to the availability of miniature and cheap spectrometers, spectral interrogation becomes a practical approach.

In this paper, a polymer waveguide coupled surface plasmon sensor for the detection of liquid with high RI by using spectral interrogation is designed. Polymer materials are widely used in the fabrication of optical waveguide devices because of easy fabrication, low cost, and good compatibility with Si fabrication technologies [30, 31]. The effects of variation of the thickness of the $\mathrm{Au}$ film, polymethyl methacrylate (PMMA) buffer, and waveguide layer on the sensing performance of the waveguide are comprehensively investigated. Numerical simulations show that in this structure, a thinner gold film gives rise to a more sensitive structure, while the variation of the thickness of the PMMA buffer and waveguide layer has a small effect on the sensitivity. For liquid with high RI, the sensitivity of the sensor increases significantly. When RI of liquid increases from 1.45 to 1.52 , a sensitivity of $4518.14 \mathrm{~nm} / \mathrm{RIU}$ is obtained, corresponding to a detection limit of $2.2 \times 10^{-7} \mathrm{RIU}$, which is higher than those of many reported works [8, 32-35]. By optimizing the geometric parameters, a high figure of merit (FOM) of 114.07 can be obtained. The designed RI sensor has potential applications in the environment, industry, and agriculture sensing fields.

\section{Structure and principle}

The designed polymer waveguide coupled surface plasmon RI sensor is sketched in Fig. 1. When the light wave is coupled into the waveguide, the waveguide mode then propagates over the metal clad region. The reflection evanescent fields will excite the SPPs when the phase matching condition is satisfied. Excitation of the SPPs is observed as a dramatic fall in the waveguide output intensity due to the lossy nature of the surface plasma waves. The variation of external RI on the metal surface will cause the change in the propagation constant of the SPP mode, and the absorption peak will change. 
Epoxy (EPO) polymer is a commonly used permanent epoxy negative photoresist. After ultraviolet (UV) exposure, its chemical stability and corrosion resistance are greatly improved due to the cross-linking solidification reaction [36]. The EPO clad and EPO core are used as the cladding layer and the waveguide core, respectively. The PMMA material is adopted as the buffer layer. The gold is used as the metal layer. The thicknesses of the waveguide, PMMA buffer, and Au layer are denoted by $h, t_{b}$, and $t_{\mathrm{Au}}$, respectively. The width of the waveguide $w$ is $5 \mu \mathrm{m}$ and the length of the Au film $L$ is $1 \mathrm{~mm}$. The RIs of the EPO clad, EPO core, PMMA, and liquid are denoted by $n_{c}, n_{o}, n_{b}$, and $n_{a}$, respectively. The index of gold $n_{\mathrm{Au}}$ varies strongly with the wavelength, taken from the experimental date [38].

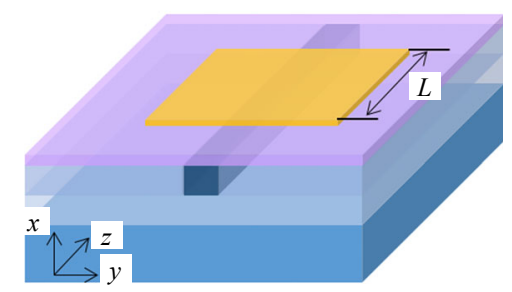

(a)

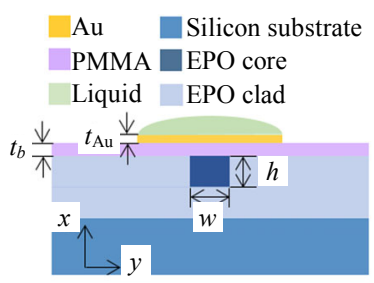

(b)

Fig. 1 Schematic diagrams of (a) 3-dimension (3-D) structure and (b) cross section of the waveguide.

\section{Simulations}

Different from coupling between the SPP waveguide and dielectric waveguide based on the coupled mode theory [27], this configuration is based on the analysis of the waveguide modes supported by the metal-clad waveguide that incurs optical losses due to the imaginary part of the effective indices [24, 37]. The sensing performance of the RI sensor is calculated by the finite difference method based on the mode expansion and propagation method. Because SPP waves are transverse magnetic (TM) polarized, only TM modes are considered in the following calculations. The RIs of the EPO clad, EPO core, PMMA are assumed to be $n_{c}=1.568, n_{o}=1.575$, and $n_{b}=1.495$, respectively [36]. Firstly, the dispersion characteristics of the individual waveguide mode and SPP mode are calculated, as shown in Fig. 2(a). The parameters are as follows: $h=5 \mu \mathrm{m}, t_{b}=500 \mathrm{~nm}$, $t_{\mathrm{Au}}=35 \mathrm{~nm}$, and $n_{a}=1$.

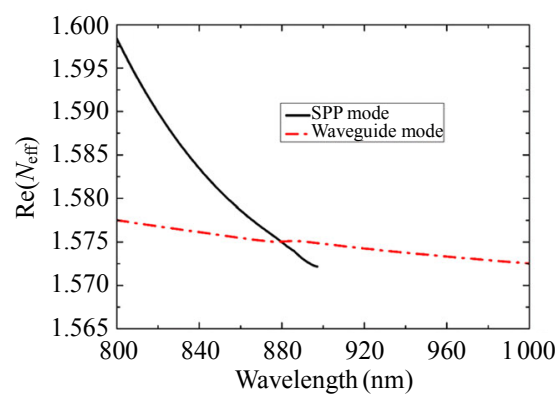

(a)

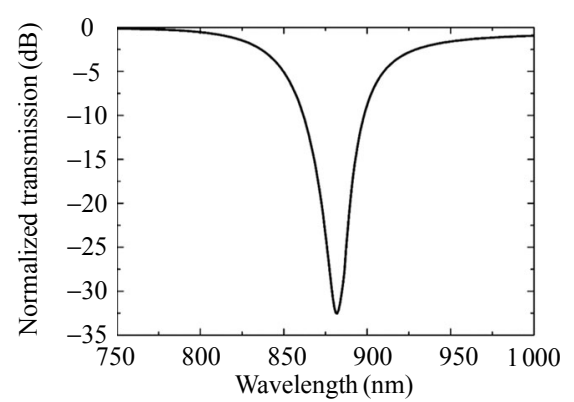

(b)

Fig. 2 Dispersion characteristics of the waveguide: (a) effective RI of waveguide mode and SPP mode and (b) normalized transmission varies with the wavelength.

The effective RI of the two modes decreases with an increase in the wavelength and intersects at $880 \mathrm{~nm}$. At this point, the phase matching condition for the SPR effect is satisfied. When the light with $880 \mathrm{~nm}$ wavelength propagates along the dielectric waveguide, the evanescent field generated in the 
cladding will excite the SPP waves on the metal surface, leading to a sharp attenuation of the optical energy in the waveguide. Figure 2(b) shows the normalized transmission variation with the wavelength. Clearly, there is a resonance with a contrast of $-33 \mathrm{~dB}$ at $880 \mathrm{~nm}$.

In order to reflect the variation of the mode field distribution, the field distributions of the mode at the non-resonance wavelength $(980 \mathrm{~nm})$ and the resonance wavelength $(880 \mathrm{~nm})$ are calculated, as shown in Fig. 3. It indicates that the mode field is mainly distributed in the dielectric waveguide at the non-resonance wavelength, while the mode field is

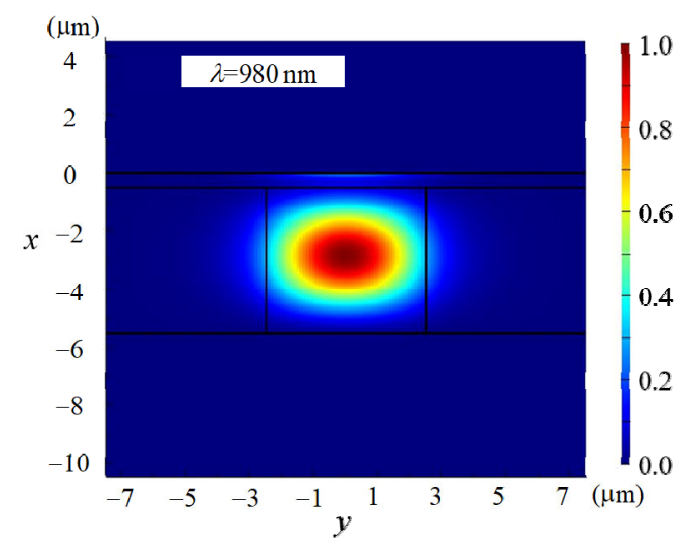

(a)

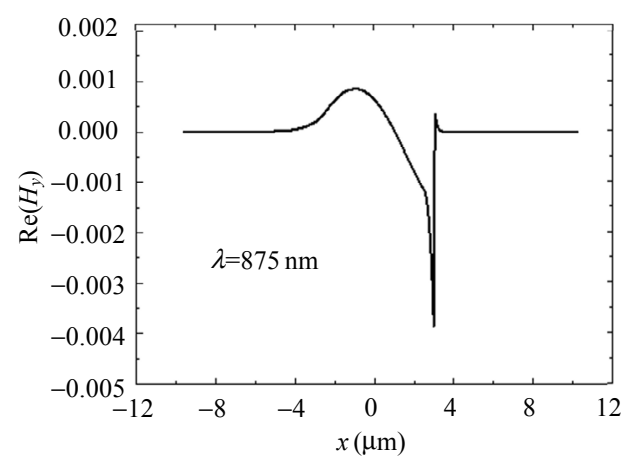

(c) mainly located in the metal layer at the resonance wavelength. Therefore, the resonance occurs at the wavelength of $880 \mathrm{~nm}$ due to the large light absorption of metal. Figures 3(c) and 3(d) show the real parts of magnetic field distribution $\operatorname{Re}\left(H_{y}\right)$ at the wavelengths of $875 \mathrm{~nm}$ and $893 \mathrm{~nm}$ (the resonance wavelength is $880 \mathrm{~nm}$ ), respectively. The SPP mode has the opposed-phase magnetic field component in the transverse plane, thus the short range SPP mode is used in the sensor. The variation of RI of liquid adjacent to the $\mathrm{Au}$ film will produce a change in the propagation constant of the SPP mode, leading to a shift in the resonance wavelength.

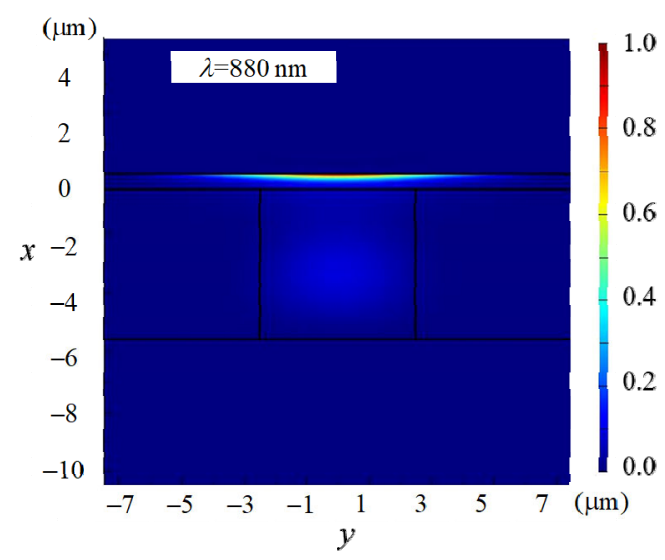

(b)

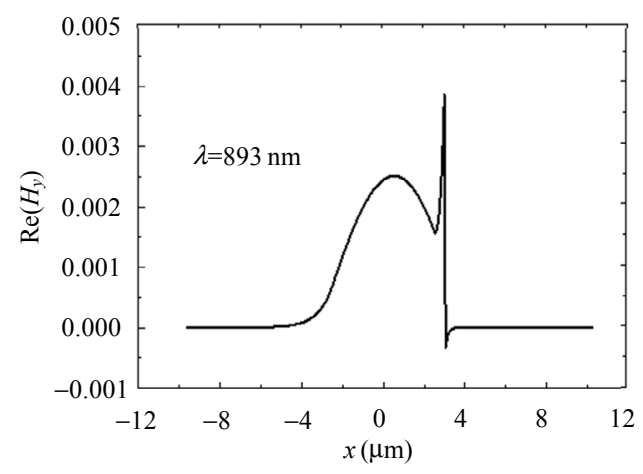

(d)

Fig. 3 Mode field distributions at the (a) non-resonant wavelength and (b) resonant waveguide; magnetic field distribution $\operatorname{Re}\left(H_{\mathrm{y}}\right)$ at the wavelength of (c) $875 \mathrm{~nm}$ and (d) $893 \mathrm{~nm}$.

Figure 4(a) shows the normalized transmission changes with the wavelength with different RIs of liquid. It can be seen that when the RI of liquid increases from 1.33 to 1.45 , the resonance wavelength has a red shift from $920 \mathrm{~nm}$ to $1060 \mathrm{~nm}$. Figure 4(b) shows the dispersion curves of the individual waveguide mode and SPP mode when the external RIs are 1 and 1.33. Clearly, when external 
RI $n_{c}$ increases to $1.33, \operatorname{Re}\left(N_{\text {eff }}\right)$ of the SPP mode increases significantly. The intersection of the waveguide mode dispersion curve and the SPP dispersion curve red shifts to $945 \mathrm{~nm}$, and a long wavelength resonance occurs. In this way, the RI of liquid can be derived by detecting the position of the resonance wavelength. Moreover, with an increase in RI of liquid from 1.33 to 1.45 , the resonance contrast increases from $-36 \mathrm{~dB}$ to $-78 \mathrm{~dB}$, since the increasing RI of liquid makes the mode field move up the waveguide structure along the $z$ direction and the TM waves will have more energy entering the metal layer. This leads to an enhancement of interaction between the mode field and metal film, and the absorption increases.

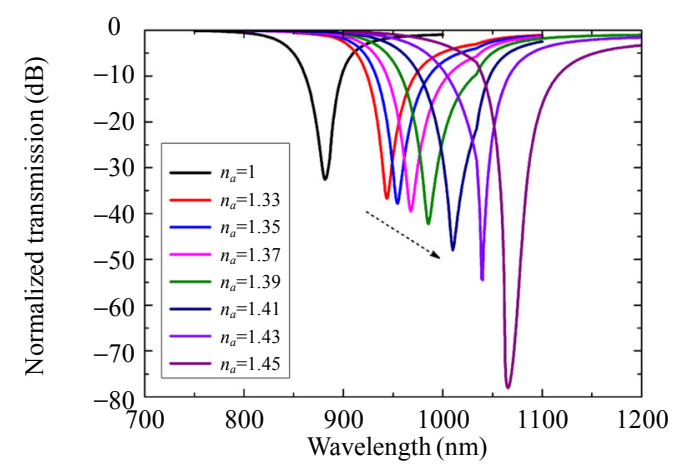

(a)

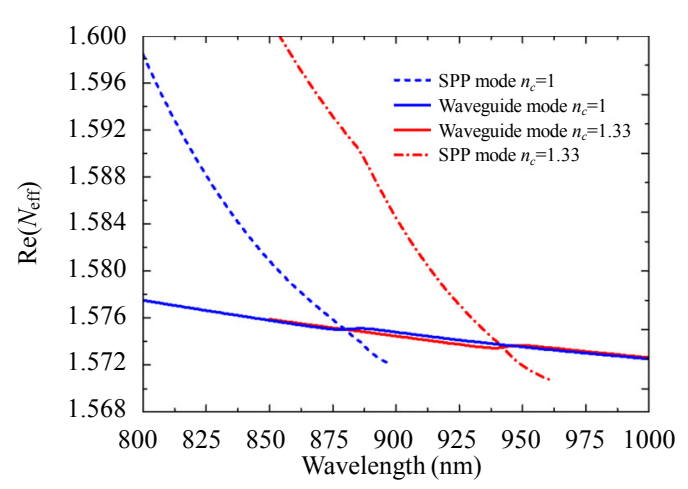

(b)

Fig. 4 Sensing characteristic to RIs of liquid: (a) normalized transmission changes with the wavelength with different RIs of liquid and (b) dispersion curves of the individual waveguide mode and SPP mode when the external RIs are 1 and 1.33.

For the spectra interrogation based SPR sensor, the sensing performance is mainly determined by the resonance wavelength, the full width of the absorption band at half height (FWHM), sensitivity, and figure of merit (FOM). The sensitivity $S$ is calculated by the ratio between the resonance wavelength variation $\Delta \lambda$ and the variation of RI of liquid $\Delta n, S=\Delta \lambda \Delta n$. The $F O M$ is calculated by

$$
F O M=\frac{S}{F W H M} .
$$

\subsection{Effects of the thickness of the Au film}

Because the thickness of the metal film directly relates to the amount of free electron charge, the variation of the thickness of the metal film on the sensing performance of the sensor is investigated. Figure 5(a) shows the normalized transmission when the thicknesses of the Au film are $35 \mathrm{~nm}, 40 \mathrm{~nm}$ and $45 \mathrm{~nm}$, respectively. The parameters are as follows: $h=5 \mu \mathrm{m}, t_{b}=500 \mathrm{~nm}$, and $n_{a}=1$. Clearly, the thickness of the metal film not only affects the resonance wavelength, but also affects the FWHM of the resonance. The FWHMs are $81.22 \mathrm{~nm}$, $72.53 \mathrm{~nm}, 56.12 \mathrm{~nm}$ for $35 \mathrm{~nm}, 40 \mathrm{~nm}$, and $45 \mathrm{~nm}$ thick gold films, respectively. Figure 5(b) shows the dispersion curves of the individual waveguide mode and SPP mode with different thicknesses of the Au film. Clearly, when the thickness of $\mathrm{Au}$ increases, the $\operatorname{Re}\left(N_{\text {eff }}\right)$ of the SPP mode decreases significantly. The intersection of dispersion curves blue shifts, and a short wavelength resonance occurs. Figure 5(c) shows the resonance wavelength varies with RI of liquid when the thicknesses of the gold film are $35 \mathrm{~nm}, 40 \mathrm{~nm}$, and $45 \mathrm{~nm}$. When the RI of liquid increases from 1.33 to 1.45 , the resonance wavelength has a red shift of $140 \mathrm{~nm}, 80 \mathrm{~nm}$, and $60 \mathrm{~nm}$ for the gold films with the thicknesses of $35 \mathrm{~nm}, 40 \mathrm{~nm}$, and $45 \mathrm{~nm}$, respectively. The corresponding average sensitivities are $1166.7 \mathrm{~nm} / \mathrm{RIU}, 666.7 \mathrm{~nm} / \mathrm{RIU}$, and $500 \mathrm{~nm} / \mathrm{RIU}$, respectively. It is obvious that a thinner gold film gives rise to a more sensitive structure. When the RI of liquid increases from 1.45 to 1.52 , the resonance wavelengths red shift by $316.27 \mathrm{~nm}, 273.52 \mathrm{~nm}$, and 
$231.14 \mathrm{~nm}$ and the sensitivities are $4518.14 \mathrm{~nm} / \mathrm{RIU}$, $3907.43 \mathrm{~nm} / \mathrm{RIU}$, and $3302 \mathrm{~nm} / \mathrm{RIU}$ for the gold film thicknesses of $35 \mathrm{~nm}, 40 \mathrm{~nm}$, and $45 \mathrm{~nm}$, respectively. It is clear that for liquid with high RI, the sensitivity of the sensor increases significantly. This is because SU-8 material with a high RI of 1.575 is used as the waveguide layer. As the RI of liquid gradually increases to approach the RI of the

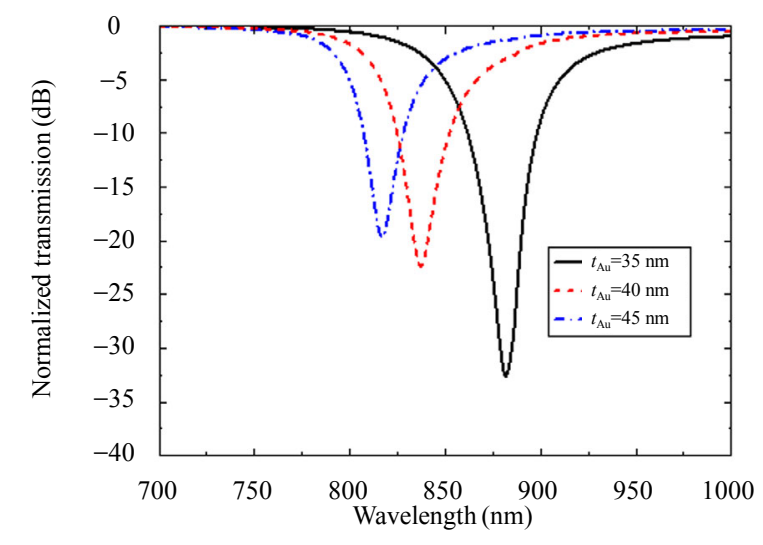

(a) waveguide layer, the TM waves will have more energy entering the metal layer, making the structure more sensitive. If the resolution of the used spectrometer is $1 \mathrm{pm}$, the RI resolution of the sensor with $35 \mathrm{~nm}$ thick $\mathrm{Au}$ film is about $2.2 \times 10^{-7} \mathrm{RIU}$. Table 1 is a list of performance parameters with different thicknesses of the Au film. The FOM can be tuned by optimizing the thickness of the $\mathrm{Au}$ film.

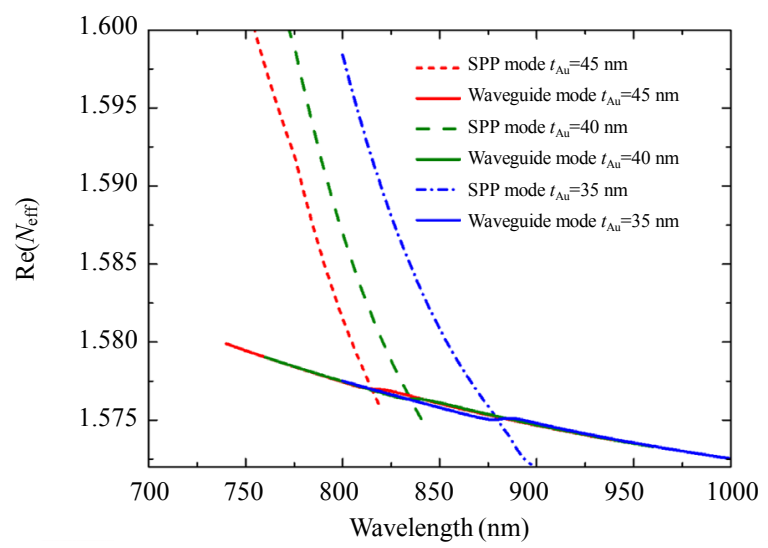

(b)

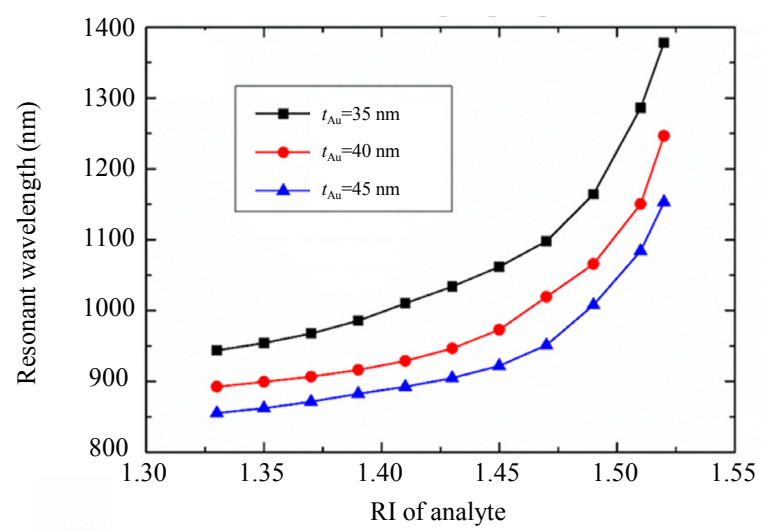

(c)

Fig. 5 Effects of the thickness of the gold film: (a) normalized transmission varies with the wavelength, (b) dispersion curves of the individual waveguide mode and SPP mode with different thicknesses of the Au film, and (c) resonance wavelength changes with the RI of liquid when the thicknesses of the gold film are $35 \mathrm{~nm}, 40 \mathrm{~nm}$, and $45 \mathrm{~nm}$.

Table 1 Performance of the SPR sensor with different thicknesses of the Au film.

\begin{tabular}{cccc}
\hline$t_{\mathrm{Au}}(\mathrm{nm})$ & Sensitivity $(\mathrm{nm} / \mathrm{RIU})$ & $\mathrm{FWHM}(\mathrm{nm})$ & $\mathrm{FOM}\left(\mathrm{RIU}^{-1}\right)$ \\
\hline 35 & 4518.14 & 81.22 & 55.63 \\
40 & 3907.43 & 72.53 & 53.87 \\
45 & 3302.00 & 56.12 & 58.83 \\
\hline
\end{tabular}

\subsection{Effects of the thickness of the PMMA buffer layer}

The thickness of the PMMA buffer layer directly affects the dispersion characteristics of the waveguide mode and the SPP mode. The influence of variation of the thickness of the buffer layer on 
the sensing performance of the sensor is investigated, as shown in Fig. 6. The structure parameters are as follows: $h=4.5 \mu \mathrm{m}, t_{\mathrm{Au}}=40 \mathrm{~nm}$, and $n_{a}=1$. The thickness of the buffer layer has a small effect on the position of the resonance wavelength, but has a great effect on the resonance intensity. When the thickness of the PMMA buffer increases from $500 \mathrm{~nm}$ to $700 \mathrm{~nm}$, the resonance wavelength slightly blue shifts from $840 \mathrm{~nm}$ to $810 \mathrm{~nm}$. The resonance intensity weakens and the resonance contrast declines from $-30 \mathrm{~dB}$ to $-4 \mathrm{~dB}$. The FWHMs are $83.14 \mathrm{~nm}, 46.07 \mathrm{~nm}$, and $33 \mathrm{~nm}$ for $500 \mathrm{~nm}, 600 \mathrm{~nm}$, and $700 \mathrm{~nm}$ thick buffer layers, respectively. This is mainly because an increase in the PMMA buffer thickness leads to an increase in the separated distance between the waveguide mode and SPP mode, and then a decrease in the coupling efficiency. Figure 6(b) shows the dispersion curves of the individual waveguide mode and SPP mode when the thicknesses of the buffer layer are $500 \mathrm{~nm}$ and $700 \mathrm{~nm}$. It is clear that when the thickness of the buffer layer increases, $\operatorname{Re}\left(N_{\text {eff }}\right)$ of the SPP mode decreases. The intersection of the waveguide dispersion curve and the SPP dispersion curve blue shifts and the resonance red shifts as decreasing $t_{b}$. Figure 6(c) shows the resonance wavelength changes with the RI of liquid when the thicknesses of the PMMA buffer layer are $500 \mathrm{~nm}, 600 \mathrm{~nm}$, and $700 \mathrm{~nm}$. It can be seen that the thickness of the buffer layer has a small effect on the sensitivity of the sensor. Similarly, for the high RI of liquid to be measured, the detection sensitivity increases significantly. When the RI of liquid increases from 1.45 to 1.52 , the resonance wavelength red shifts by $273.43 \mathrm{~nm}$, $272.89 \mathrm{~nm}$, and $263.496 \mathrm{~nm}$, and the corresponding sensitivities are $3906.14 \mathrm{~nm} / \mathrm{RIU}, 3898.43 \mathrm{~nm} / \mathrm{RIU}$, and $3764.23 \mathrm{~nm} / \mathrm{RIU}$ for the PMMA buffer thicknesses of $500 \mathrm{~nm}, 600 \mathrm{~nm}$, and $700 \mathrm{~nm}$, respectively. When the thickness of the buffer layer increases, the sensitivity of the sensor slightly decreases.

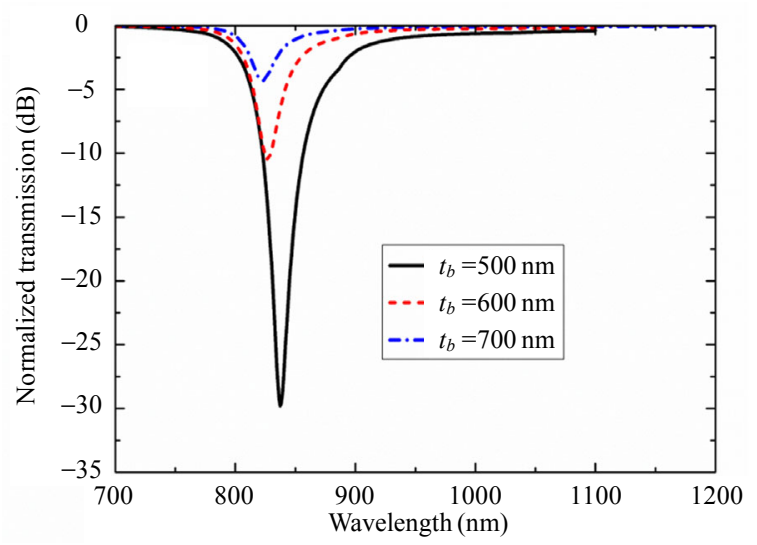

(a)

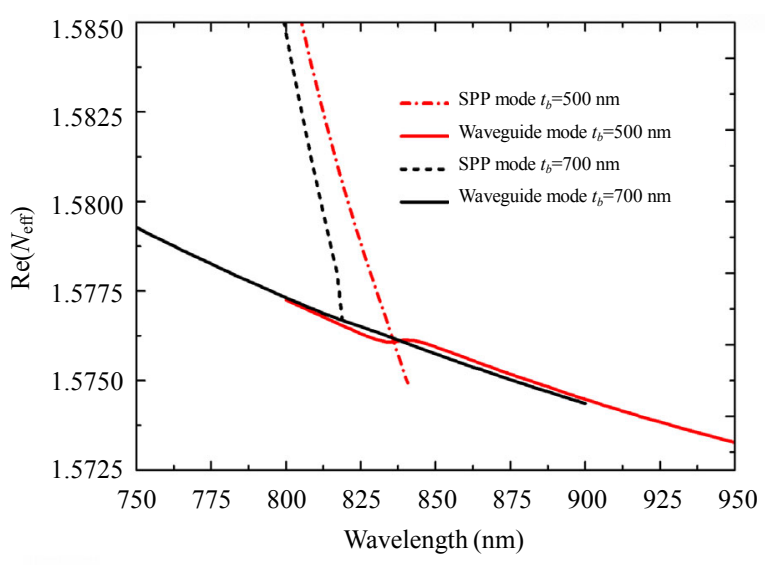

(b)

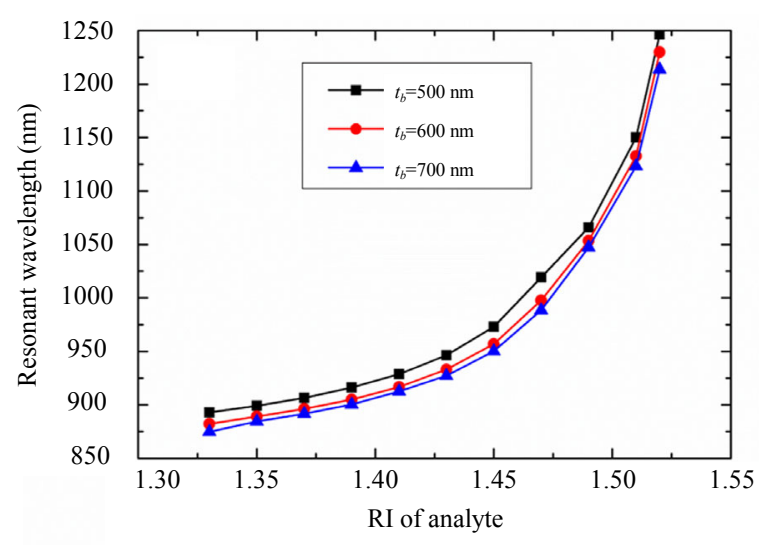

(c)

Fig. 6 Effects of the thickness of the PMMA buffer layer: (a) normalized transmission varies with the wavelength, (b) dispersion curves of the individual waveguide mode and SPP mode when the thicknesses of the buffer layer are $500 \mathrm{~nm}$ and $700 \mathrm{~nm}$, and (c) resonance wavelength changes with RI of liquid for different PMMA buffer thicknesses.

Table 2 is a list of performance parameters with different thicknesses of the PMMA buffer layer. Due to the decrement of FWHM, FOM increases from 
46.98 to 114.07 when the buffer thickness increases from $500 \mathrm{~nm}$ to $700 \mathrm{~nm}$.

Table 2 Performance of the SPR sensor with different thicknesses of the buffer layer.

\begin{tabular}{cccc}
\hline$t_{b}(\mathrm{~nm})$ & Sensitivity $(\mathrm{nm} / \mathrm{RIU})$ & $\mathrm{FWHM}(\mathrm{nm})$ & $\mathrm{FOM}\left(\mathrm{RIU}^{-1}\right)$ \\
\hline 500 & 3906.14 & 83.14 & 46.98 \\
600 & 3898.43 & 46.07 & 84.62 \\
700 & 3764.23 & 33.00 & 114.07 \\
\hline
\end{tabular}

\subsection{Effects of the thickness of the waveguide}

Figure 7(a) shows the normalized transmission when the thicknesses of the waveguide are $4.5 \mu \mathrm{m}$, $5 \mu \mathrm{m}$, and $5.5 \mu \mathrm{m}$. The parameters are as follows: $t_{b}=$ $500 \mathrm{~nm}, t_{\mathrm{Au}}=40 \mathrm{~nm}$, and $n_{a}=1$. It can be seen that with an increase in the waveguide thickness, the resonance wavelength does not change notably, but the resonance intensity is obviously weakened. When the thickness of the waveguide increases from $4.5 \mu \mathrm{m}$ to $5.5 \mu \mathrm{m}$, the resonance contrast declines from $-30 \mathrm{~dB}$ to $-17 \mathrm{~dB}$. FWHMs are $82.97 \mathrm{~nm}$, $72.77 \mathrm{~nm}$, and $62.85 \mathrm{~nm}$ for $4.5 \mu \mathrm{m}, 5 \mu \mathrm{m}$, and $5.5 \mu \mathrm{m}$ thick waveguide layers, respectively. Because a thinner waveguide gives rise to an increase in the electric field density in the waveguide, as well as an increase in the mode field overlap between the dielectric waveguide mode and SPP mode. Figure 7(b) shows the calculated dispersion characteristics of the individual waveguide mode and SPP mode when the thicknesses of the waveguide layer are $4.5 \mu \mathrm{m}$ and $5.5 \mu \mathrm{m}$, respectively. Clearly, when the thickness of the waveguide layer increases, $\operatorname{Re}\left(N_{\text {eff }}\right)$ of the waveguide mode increases while that of the SPP mode almost has no change. A slight blue shift of the intersection of the dispersion curves occurs as increasing $h$. Figure 7(c) shows the resonance wavelength changes with the RI of liquid with different thicknesses of the waveguide layer. The thickness of the waveguide layer has little effect on the sensitivity. Table 3 is a list of performance parameters with different thicknesses of the waveguide layer. Due to the decrement of FWHM, FOM increases from 47.87 to 62.19 when the waveguide thickness increases from $5.0 \mu \mathrm{m}$ to $5.5 \mu \mathrm{m}$.

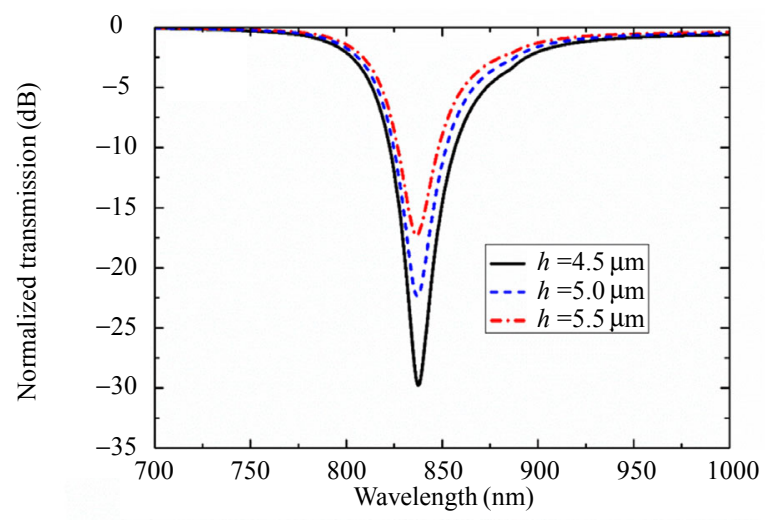

(a)

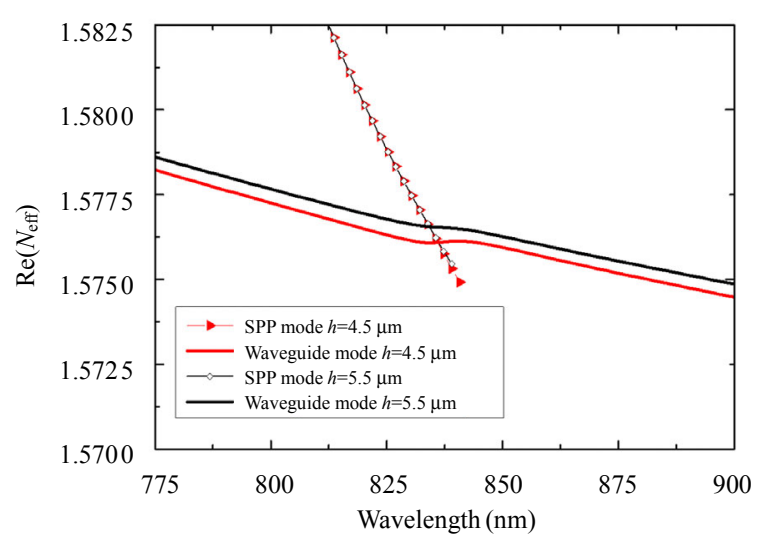

(b)

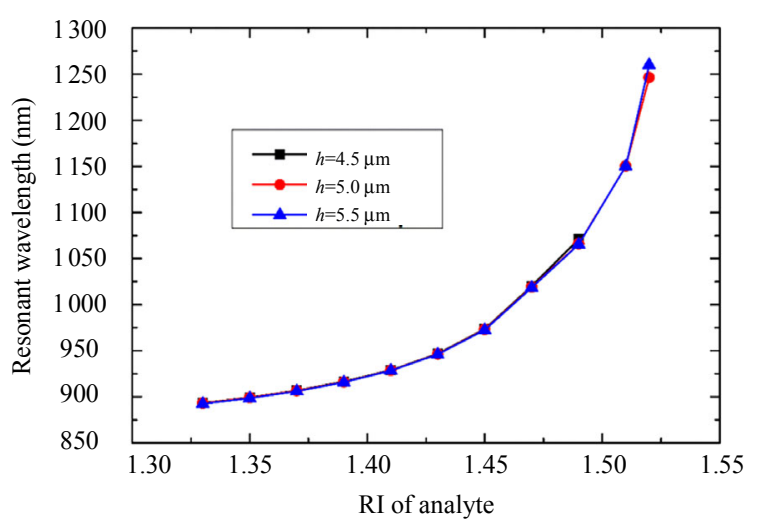

(c)

Fig. 7 Effects of the thickness of the waveguide: (a) normalized transmission varies with the wavelength, (b) dispersion curves of the individual waveguide mode and SPP mode when the thicknesses of the waveguide layer are $4.5 \mu \mathrm{m}$ and $5.5 \mu \mathrm{m}$, and (c) the resonance wavelength changes with the RI of liquid for different waveguide thicknesses. 
Table 3 Performance of the SPR sensor with different thicknesses of the waveguide layer.

\begin{tabular}{cccc}
\hline$h(\mu \mathrm{m})$ & Sensitivity $(\mathrm{nm} / \mathrm{RIU})$ & FWHM $(\mathrm{nm})$ & $\mathrm{FOM}\left(\mathrm{RIU}^{-1}\right)$ \\
\hline 4.5 & $\mathrm{NA}$ & 82.97 & NA \\
5.0 & 3907.43 & 78.35 & 49.87 \\
5.5 & 3904.24 & 62.85 & 62.19 \\
\hline
\end{tabular}

To illustrate the characteristics of the proposed design, key parameters of the SPR-based RI sensors are compared with those in some reported works [39-42]. As shown in Table 4, the designed SPR sensor in this work has a balanced performance of high sensitivity of 3764.23 and high FOM of 114.07 .

Table 4 Performance comparison between SPR-based RI sensors.

\begin{tabular}{ccccc}
\hline Description & RI range & Sensitivity & FWHM & $\begin{array}{c}\text { FOM } \\
\left(\mathrm{RIU}^{-1}\right)\end{array}$ \\
\hline $\begin{array}{c}\text { Gold nanograting } \\
\text { [39] }\end{array}$ & $1.0-1.1$ & $317 \mathrm{~nm} / \mathrm{RIU}$ & $38 \mathrm{~nm}$ & 8.0 \\
$\begin{array}{c}\text { Gold nano disk array } \\
\text { [40] }\end{array}$ & $1.3-1.4$ & $853 \mathrm{~nm} / \mathrm{RIU}$ & $9 \mathrm{~nm}$ & 126.0 \\
$\begin{array}{c}\text { SPR sensor with } \\
\text { absentee layer [41] }\end{array}$ & $1.5-5.0$ & $5.4\left(^{\circ}\right) / \mathrm{RIU}$ & $0.313^{\circ}$ & 17.2 \\
$\begin{array}{c}\text { Long-range surface } \\
\text { plasmon-based fiber } \\
\text { sensor [42] }\end{array}$ & $1.33-1.38$ & $3628 \mathrm{~nm} / \mathrm{RIU}$ & $34 \mathrm{~nm}$ & 106.7 \\
This work & $1.45-1.52$ & $3764 \mathrm{~nm} / \mathrm{RIU}$ & $33 \mathrm{~nm}$ & 114.1 \\
\hline
\end{tabular}

\section{Conclusions}

A waveguide-type SPP sensor for the detection of liquid with high refractive index is designed based on polymer materials. The effects of variation of the thickness of the Au film, PMMA buffer, and waveguide layer on the sensing performance of the waveguide are comprehensively investigated by using the finite difference method. Numerical simulations show that in this structure, a thinner gold film gives rise to a more sensitive structure, while the variation of the thickness of the PMMA buffer and waveguide layer has a small effect on the sensitivity. But FOM of the sensor can be tuned effectively by the thicknesses of the PMMA buffer and waveguide layer. When RI of liquid increases from 1.45 to 1.52 , the sensitivity reaches
$4518.14 \mathrm{~nm} / \mathrm{RIU}$. The corresponding detection resolution is about $2.2 \times 10^{-7} \mathrm{RIU}$. By optimizing the geometric parameters, the FOM of 114.07 can be obtained. The waveguide coupled surface plasmon RI sensor is compact, low-cost, and high integration density, and shows potential applications in combining SPR sensor with the absorption spectroscopy and in the fields of environment, industry, and agriculture sensing.

\section{Acknowledgment}

The authors gratefully acknowledge the financial support from the Shandong Provincial Key Research and Development Program (Grant Nos. 2018YFJH0702 and 2019JZZY020711) and Qingdao Postdoctoral Applied Research Project (Grant No. 6242007311086).

Open Access This article is distributed under the terms of the Creative Commons Attribution 4.0 International License (http://creativecommons.org/licenses/by/4.0/), which permits unrestricted use, distribution, and reproduction in any medium, provided you give appropriate credit to the original author(s) and the source, provide a link to the Creative Commons license, and indicate if changes were made.

\section{References}

[1] M. S. Hoseinian and M. A. Bolorizadeh, "Design and simulation of a highly sensitive SPR optical fiber sensor," Photonic Sensors, 2019, 9(1): 33-42.

[2] A. Motogaito and Y. Ito, "Excitation mechanism of surface plasmon polaritons for surface plasmon sensor with 1d metal grating structure for high refractive index medium," Photonic Sensors, 2019, 9(1): 11-18.

[3] E. Ozbay, "Plasmonics: Merging photonics and electronics at nanoscale dimensions," Science, 2006, 311(5758): 189-193.

[4] J. Homola, "Surface plasmon resonance sensors for detection of chemical and biological species," Chemical Reviews, 2008, 108(2): 462-493.

[5] Jiř́ Homola, Sinclair S. Yee, and Günter Gauglitz, "Surface Plasmon Resonance Sensors: Review," Analytical \& Bioanalytical Chemistry, 1999, 377(3): 528-539.

[6] Y. Yuan, N. Yuan, D. Gong, and M. H. Yang, “A high-sensitivity and broad-range SPR glucose sensor 
based on improved glucose sensitive membranes," Photonic Sensors, 2019, 9(4): 309-316.

[7] G. Xiao and H. Y. Yang, "Modeling of refractive index sensing using Au aperture arrays on a Bragg fiber facet," Photonic Sensors, 2019, 9(4): 337-343.

[8] W. R. Wong, O. Krupin, F. R. Mahamd Adikan, and P. Berini, "Optimization of long-range surface plasmon waveguides for attenuation-based biosensing," Journal of Lightwave Technology, 2015, 33(15): 3234-3242.

[9] W. R. Wong, S. D. Sekaran, F. R Adikan, and P. Berini, "Detection of dengue NS1 antigen using long-range surface plasmon waveguides," Biosensors and Bioelectronics, 2016, 78: 132-139.

[10] O. Krupin, H. Asiri, C. Wang, R. N. Tait, and P. Berini, "Biosensing using straight long-range surface plasmon waveguides," Optics Express, 2013, 21(1): 698-709.

[11] D. C. Li, B. Y. Lu, R. Zhu, H. X. Yu, and K. X. Xu, "An optofluidic system with volume measurement and surface plasmon resonance sensor for continuous glucose monitoring," Biomicrofluidics, 2016, 10(1): 011913.

[12] S. K. Vashist, E. M. Schneider, and J. H. T. Luong, "Surface plasmon resonance-based immunoassay for human C-reactive protein," Analyst, 2015, 140(13): 4445-4452.

[13] A. Aray, F. Chiavaioli, M. Arjmand, C. Trono, S. Tombelli, A. Giannetti, et al., "SPR-based plastic optical fibre biosensor for the detection of C-reactive protein in serum," Journal of Biophotonics, 2016, 9(10): 1077-1084.

[14] L. Wu, J. Guo, H. Xu, X. Dai, and Y. Xiang, "Ultrasensitive biosensors based on long-range surface plasmon polariton and dielectric waveguide modes," Photonics Research, 2016, 4(6): 262-266.

[15] D. Michel, F. Xiao, and K. Alameh, "A compact, flexible fiber-optic surface plasmon resonance sensor with changeable sensor chips," Sensors and Actuators B: Chemical, 2017, 246: 258-261.

[16] M. Vala, S. Etheridge, J. A. Roach, and J. Homola, "Long-range surface plasmons for sensitive detection of bacterial analytes," Sensors and Actuators B: Chemical, 2009, 139(1): 59-63.

[17] Q. Liu and K. S. Chiang, "Refractive-index sensor based on long-range surface plasmon mode excitation with long-period waveguide grating," Optics Express, 2009, 17(10): 7933-7942.

[18] J. Dostálek, J. Čtyrokýa, J. Homola, E. Brynda, M. Skalsky, P. Nekvindová, et al., "Surface plasmon resonance biosensor based on integrated optical waveguide," Sensors and Actuators B: Chemical, 2001, 76(1-3): 8-12.

[19] A. K. Mishra, S. K. Mishra, and B. D. Gupta, "SPR based fiber optic sensor for refractive index sensing with enhanced detection accuracy and figure of merit in visible region," Optics Communications, 2015,
344: 86-91.

[20] A. K. Sheridan, P. N. Ngamukot, P. N. Bartlett, and J. S. Wilkinson, "Waveguide surface plasmon resonance sensing: Electrochemical desorption of alkane thiol monolayers," Sensors and Actuators B: Chemical, 2006, 117(1): 253-260.

[21] Z. Jin, W. Guan, C. Liu, T. Xue, Q. Wang, W. Zheng, et al., "A stable and high resolution optical waveguide biosensor based on dense $\mathrm{TiO}_{2} / \mathrm{Ag}$ multilayer film," Applied Surface Science, 2016, 377 : 207-212.

[22] D. V. Nesterenko, S. Hayashi, and Z. Sekkat, "Extremely narrow resonances, giant sensitivity and field enhancement in low-loss waveguide sensors," Journal of Optics, 2016, 18(6): 065004.

[23] C. R. Lavers and J. S. Wilkinson, "A waveguide-coupled surface-plasmon sensor for an aqueous environment," Sensors and Actuators B: Chemical, 1994, 22(1): 75-81.

[24] R. D. Harris and J. S. Wilkinson, "Waveguide surface plasmon resonance sensors," Sensors and Actuators B: Chemical, 1995, 29(1-3): 261-267.

[25] C. Mouvet, R. D. Harris, C. Maciag, B. J. Luff, and G. Ismail, "Determination of simazine in water samples by waveguide surface plasmon resonance," Analytica Chimica Acta, 1997, 338(1-2): 109-117.

[26] M. N. Weiss and R. Srivastava, "Experimental investigation of a surface plasmon-based integrated-optic humidity sensor," Electronics Letters, 1996, 32(9): 842-843.

[27] F. Liu, R. Wan, Y. Huang, and J. Peng, "Refractive index dependence of the coupling characteristics between long-range surface-plasmon-polariton and dielectric waveguide modes," Optics Letters, 2009, 34(17): 2697-2699.

[28] B. Fan, L. Fang, Y. Li, X. Wang, K. Cui, F. Xue, et al., "Integrated refractive index sensor based on hybrid coupler with short range surface plasmon polariton and dielectric waveguide," Sensors and Actuators B: Chemical, 2013, 186: 495-505.

[29] L. Ji, X. Sun, G. He, L. Yu, X. Wang, Y. Yi, et al., "Surface plasmon resonance refractive index sensor based on ultraviolet bleached polymer waveguide," Sensors and Actuators B: Chemical, 2017, 244: 373-379.

[30] S. K. Mishra, B. Zou, and K. S. Chiang, "Surface-plasmon-resonance refractive index sensor with $\mathrm{Cu}$ coated polymer waveguide," IEEE Photonics Technology Letters, 2016, 28(17): 1835-1838.

[31] I. C. Liu, P. C. Chen, L. K. Chau, and G. E. Chang, "Optofluidic refractive-index sensors employing bent waveguide structures for low-cost, rapid chemical and biomedical sensing," Optics Express, 2018, 26(1): 273-283.

[32] A. K. Sharma and B. D. Gupta, "Fiber optic sensor based on surface plasmon resonance with 
nanoparticle films," Photonics and Nanostructures-Fundamentals and Applications, 2005, 3(1): 30-37.

[33] M. Kanso, S. Cuenot, and G. Louarn, "Sensitivity of optical fiber sensor based on surface plasmon resonance: modeling and experiments," Plasmonics, 2008, 3(2-3): 49-57.

[34] Y. S. Dwivedi, A. K. Sharma, and B. D. Gupta, "Influence of design parameters on the performance of a surface plasmon sensor based fiber optic sensor," Plasmonics, 2008, 3(2-3): 79-86.

[35] R. K. Verma, A. K. Sharma, and B. D. Gupta, "Surface plasmon resonance based tapered fiber optic sensor with different taper profiles," Optics Communications, 2008, 281(6): 1486-1491.

[36] G. B. He, Y. Gao, Y. Xu, L. T. Ji, X. Q. Sun, X.B. Wang, Y. J. Yi, C. M. Chen, F. Wang, D. M. Zhang and $\mathrm{Y}$. D. Wu, "Design and fabrication of three-dimensional polymer mode multiplexer based on asymmetric waveguide couplers," Journal of Optics, 2018, 20: 055801.

[37] J. N. Polky and G. L. Mitchell, "Metal-clad planar dielectric waveguide for integrated optics," Journal of the Optical Society of America, 1974, 64(3): 274-279.
[38] J. Cw. Ingram, K. W. Nebesny, and J. E. Pemberton, "Optical constants of the noble metals determined by reflection electron energy loss spectroscopy," Applied Surface Science, 1990, 44(4): 293-300.

[39] Z. Y. Zhang, L. N. Wang, H. F. Hu, K. W. Li, X. P. $\mathrm{Ma}$, and G. F. Song, "A high figure of merit localized surface plasmon sensor based on a gold nanograting on the top of a gold planar film," Chinese Physics B, 2013, 22(10): 104213.

[40] X. Wang, J. K. Zhu, H. Tong, X. D. Yang, X. Wu, Z. Y. Pang, et al., "A theoretical study of a plasmonic sensor comprising a gold nano-disk array on gold film with a $\mathrm{SiO}_{2}$ spacer," Chinese Physics B, 2019, 28(4): 044201.

[41] Q. Q. Meng, X. Zhao, S. J. Chen, C. Y. Lin, Y. C. Ding, and Z. Y. Chen, "Performance analysis of surface plasmon resonance sensor with high-order absentee layer," Chinese Physics B, 2017, 26(12): 124213.

[42] X. Feng, M. Yang, Y. Luo, J. Tang, H. Guan, J. Fang, et al., "Long range surface plasmon resonance sensor based on side polished fiber with the buffer layer of magnesium fluoride," Optical and Quantum Electronics, 2017, 49(4): 147. 